NENA $\sqrt{4}$

ANIIU
CANADIAN JOURNAL of EMERGENCY NURSING

JOURNAL CANADIEN des INFIRMIÈRES D'URGENCE

THE OFFICIAL JOURNAL OF THE NATIONAL EMERGENCY NURSES' ASSOCIATION

WWW.NENA.ca

\title{
Emergency Nursing Certification in Canada
}

\section{Heather McLellan, MEd, BN, RN, CEN, CFRN}

S pecialty nursing certifications recognize mastery of clinical and theoretical knowledge, clinical judgement, and experience within a specific nursing specialty. The Canadian Nurses Association offers a specialty certification credentialing exam for registered nurses. There are also certification programs available in other countries, such as the Board of Certification for Emergency Nursing in the United States. However, the focus of this article will be about the Canadian experience.

\section{Why is certification important?}

Becoming certified in your specialty indicates to your patients, employers, and colleagues that you have mastered a core set of theoretical concepts specific to emergency nursing. This can help you in delivering safe, efficient, and high-quality patient care. Some studies have shown that certified nurses have a positive effect on patient outcomes (Krapohl et al., 2012). In addition, being certified may help you advance your career and have positive financial effects in terms of stipends.

\section{What are the eligibility criteria for becoming certified?}

The exam's eligibility criteria include holding an active licence as a registered nurse and meeting the specific experience and/

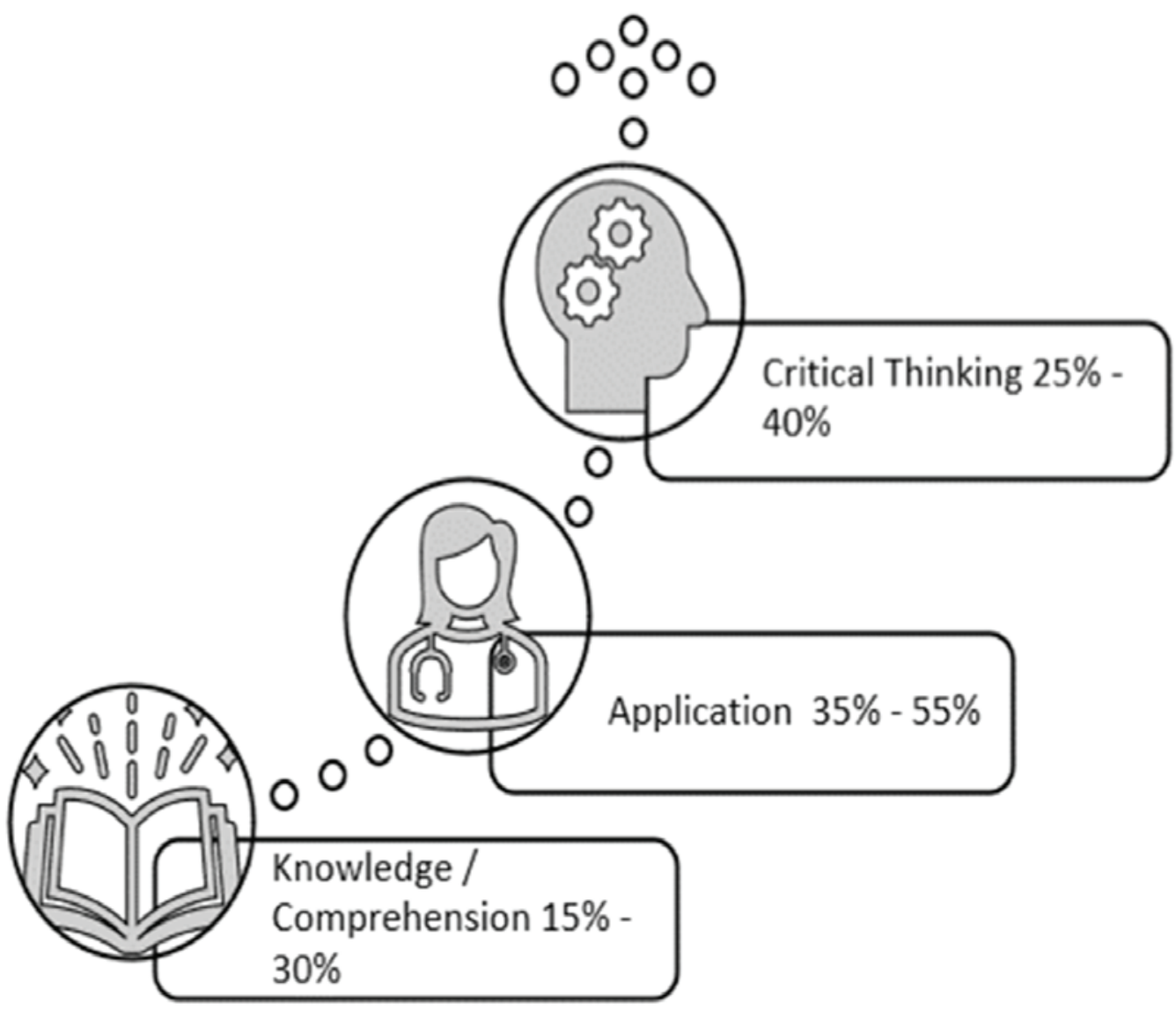


or education expectations. The experience criteria option stipulates that the candidate must have a minimum of 1,950 hours over the past five years in the specialty area. If using the combined experience and education option to demonstrate eligibility, you will need to (a) have taken a formal post-basic specialty course from a community college or university that is at least 300 hours in length and (b) have a minimum of 1,000 hours of clinical experience in your specialty over the last five years.

\section{What content is covered on the emergency nursing certification exam?}

The exam is based on the NENA Emergency Nursing Scope and Standards of Canadian Practice Sixth Edition (NENA, 2018) and the ENC(C) Exam Blueprint (CNA, 2020).

The exam consists of approximately 165 questions with 21 categories of topics related to both clinical emergencies and professional issues. These questions are distributed across three levels of cognitive response. Knowledge and Comprehension, which is a more basic level of question requiring recall of knowledge and facts; Application, in which you must take information that you know and apply it in a specific situation, and finally Critical Thinking, which is a higher level of question requiring you to analyze information and extrapolate beyond the immediate information and to identify priorities in managing a situation.

Finally, the topics are sorted by age, client culture, client health situation, and health-care environment. A comprehensive breakdown of the exam blueprint, topic categories, and competency examples can be found on the CNA website (see the link at the end of this article).

\section{How do I prepare for the exam?}

NENA and CJEN want to help you prepare for this exam. NENA supports its members by offering access to a bursary to support both those writing the certification exam for the first time or those renewing their certification. Preparation resources for NENA members include access to a free online preparation program based on the CNA's Emergency Nursing Examination Blueprint document (see NENA link below for more information).

The editors at CJEN are pleased to introduce a new section in this issue of the journal featuring certification exam sample questions with answers and rationales. We want to thank our two ENC(C) mentors and item writers, Margaret Dymond and Leanne Tyler, who helped produce this section by authoring and editing these questions to align with the current expectations of the exam. We look forward to the inclusion of this regular feature as a way of supporting Canadian emergency nurses on their journey to certification.

\section{Links and resources:}

Canadian Nurses Association (CNA): https://www. cna-aiic.ca/en/certification/exam-preparation/ exam-competencies-and-blueprints

National Emergency Nurses Association (NENA): https://nena. ca/courses/

\section{References}

Canadian Nurses Association (CNA). (2020). Emergency nursing: Exam blueprint and specialty competencies. https://www.cnaaiic.ca/-/media/nurseone/files/en/emergency_blueprint_e. pdf ? la = en \&hash $=0628371$ D 03 AC $05849206217 \overline{3} 2$ B3A53D702F33D2E

Krapohl, G., Mananojlovich, M., Reman, R. \& Zhang, L. (2012). Does certification of staff nurses improve patient outcomes?
Evidence-based Nursing, 15(2), 54-55. http://doi.org/10.1136/ ebnurs.2011.100228

National Emergency Nurses Association (NENA). (2018). Emergency nursing:Scopeandstandards of Canadianpractice (6thed.). Retrieved from https://nena.ca/standards-of-ed-nursing-practice/ 